Supporting Information for

\title{
Machine learning predictions of high arsenic and high manganese at drinking water depths of the glacial aquifer system, northern continental United States
}

\author{
Melinda L. Erickson ${ }^{1}$, Sarah M. Elliott ${ }^{1}$, Craig J. Brown ${ }^{2}$, Paul E. Stackelberg ${ }^{3}$, Katherine M. \\ Ransom ${ }^{4}$, James E. Reddy, and Charles A. Cravotta III \\ 1U.S. Geological Survey, 2280 Woodale Drive, Mounds View, MN, 55112, United States \\ 2U.S. Geological Survey, 101 Pitkin Street, East Hartford, CT, 06108, United States \\ ${ }^{3}$ U.S. Geological Survey, 425 Jordan Road, Troy, NY 12180, United States \\ ${ }^{4}$ U.S. Geological Survey, 6000 J St, Sacramento, CA 95819, United States \\ 5 U.S. Geological Survey, 30 Brown Road, Ithaca, New York 14850, United States \\ ${ }^{6}$ U.S. Geological Survey, 215 Limekiln Road, New Cumberland, PA, 17070, United States
}

\section{Contents of this file, 14 pages}

Table S1 to S6 captions. Tables uploaded separately (Excel tables).

Methods

Figures $\mathrm{S} 1$ to $\mathrm{S} 8$

\section{Additional Supporting Information (1 file with 6 tabs, uploaded separately)}

Table S1. Generalized characteristics of Quaternary sediments in the map units within hydrogeologic terranes in the glaciated area of the conterminous U.S. Table S2. Summary of data used to predict the probabilities of high arsenic and high manganese across the U.S. glacial aquifer system.

Table S3. Predictor variables included in boosted regression tree (BRT) model development. Variables retained after recursive feature elimination (RFE, as described in the Methods section) were used for predicting the likelihood of high arsenic and high manganese, and then making representative maps.

Table S4. Performance metrics for cross validation (CV), one standard error (1SE), and recursive feature elimination (RFE) models predicting the occurrence of high arsenic or high manganese.

Table S5. Relative influence of predictor variables in Boosted Regression Tree (BRT) models of high-As and high-Mn.

Table S6. Uncertainty category based on predicted probability of high As, predicted probability of high $\mathrm{Mn}$, and prediction interval. 


\section{Introduction}

This supporting information provides additional detail about machine learning models, the hydrogeologic setting of the glacial aquifer system (GLAC), response and predictor variables used to create the boosted regression tree (BRT) model, statistical model development methods, and additional tables and figures of model results. Arsenic (As) and manganese $(\mathrm{Mn})$ are commonly found at concentrations exceeding drinking water thresholds in the GLAC ${ }^{1}$. Any use of trade, firm, or product names is for descriptive purposes only and does not imply endorsement by the U.S. Government.

Machine learning $(\mathrm{ML})$ models are being used in environmental sciences to predict redox conditions, $\mathrm{pH}$, and contaminants in groundwater $\mathrm{r}^{2-9}$. $\mathrm{ML}$ techniques do not determine causative factors directly, but they can give better predictive accuracy than more traditional models ${ }^{10}$. Other benefits of $\mathrm{ML}$ models include the ability to handle predictor variables with missing values, to incorporate complex interactions between predictor variables automatically, to use data of different types (i.e., numerical, categorical, etc.), and to use data that do not meet specific data distribution assumptions ${ }^{11}$. ML models, such as BRT, highlight variables that are most strongly related to a response variable of interest. Although ML models do not provide traditional statistics related to specific important predictor variables, they do include useful measures of relative importance and direction of influence of predictor variables on the response variable ${ }^{12}$. Predictive models of high arsenic (As) and high manganese (Mn) in the Central Valley aquifer, CA, U.S., demonstrate that readily available data sources permit development of models with reasonable predictive power, and the models show differences in predicted likelihood of high concentrations at specified domestic and public supply well depths ${ }^{4,13}$. The probability of high As was predicted at typical domestic well depths for wells with typical well construction characteristics in glacial aquifers in the north-central U.S. ${ }^{5} \mathrm{~A}$ recent model presents likelihood of As concentrations exceeding specified concentrations thresholds at domestic well depths within any aquifer across the contiguous U.S. ${ }^{14}$

\section{Methods}

Approximately $75 \%$ of sites had one measurement; if more than one measurement was available for a site, the most recent value was included in analyses. This choice enforces equity for each site: each site is 'represented' by its most recent measurement. At $>90 \%$ of the sites with more than one value, all measured values were in the same model category (As $\leq 10 \mu \mathrm{g} / \mathrm{L}$ or As $>10 \mu \mathrm{g} / \mathrm{L} ; \mathrm{Mn} \leq 300 \mu \mathrm{g} / \mathrm{L}$ or $\mathrm{Mn}>300 \mu \mathrm{g} / \mathrm{L}$ ).

We used BRT with a Bernoulli distribution, appropriate for threshold models, to produce 3-dimensional probabilities of elevated $\mathrm{As}(>10 \mu \mathrm{g} / \mathrm{L})$ and $\mathrm{Mn}(>300 \mu \mathrm{g} / \mathrm{L})$ across the GLAC. Analysis was completed with the gbm package $\left(v .2 .1 .5^{15}\right)$ using R v. 4.0.0 in RStudio v. 1.2.5042 ${ }^{16}$. The BRT method combines a large number of regression tree models by focusing on the unexplained error in previous trees ${ }^{2,4,11}$, consequently minimizing the loss function and providing an optimized model. Different combinations 
of the following model tuning parameters were considered to assess predictive performance of models with varying complexity: tree complexity $\left(t_{c}\right)$ [interaction depth, or the number of interactions allowed in each tree]; shrinkage parameter $\left(l_{r}\right)$ [the fraction that each tree contributes to the total prediction]; and number of trees (n.trees). Combinations of tuning parameters were evaluated by varying $t_{c}$ from 2 to 18 by steps of $2 ; I_{r}$ from 0.002 to 0.014 by steps of 0.002 ; and n.trees from 500 to 5000 by steps of 500 . A fourth parameter, number of observations per tree terminal node, was held constant at 10 in our models to reduce memory requirements and model runtimes. More complex models tend to have high $t_{c}$ and $I_{r}$, with fewer number of trees, compared to simpler models.

Because BRT can identify complex relations among variables, it is prone to overfitting. Overfitted models tend to be very complex and do not predict well to new data so we performed 10 -fold cross validation ${ }^{12,17}$ using the caret package (v. $\left.6.0-86^{18}\right)$ on the model training dataset. For both $\mathrm{As}$ and $\mathrm{Mn}$, data were divided into training and testing datasets for model training and predictive performance evaluation, respectively. Testing data consisted of a random subset of $20 \%$ of the original dataset. A total of 630 cross validation tuned models were evaluated to identify the model with highest accuracy for the $\mathrm{Mn}$ model and highest sensitivity for the As model. The 10 -fold cross validation divides the training data into 10 groups, then trains models of varying complexity while withholding $1 / 10$ th of the data for validation purposes ${ }^{17}$. Results from cross validation were used to identify the model with the highest accuracy $(\mathrm{Mn})$ or highest sensitivity (As); these models are referred to throughout as the 'CV models'.

A simpler model (i.e. lower $t_{c}$, lower $I_{r}$, and more trees) with total accuracy or sensitivity within one standard error of the CV model was identified. The simpler one standard error (1SE) model can generalize to new data better than the CV model without sacrificing predictive power, and in some cases can perform better than the CV model. The 1SE Mn model resulting in a combination of the highest accuracy and highest predicted prevalence was chosen from a suite of 149 models. Although the 1SE As model was chosen similarly, we took some extra steps to account for bias that may be introduced across the GLAC because of the imbalance dataset. We predicted the probability of high As for all 66 1SE models using the testing dataset. We then used those predictions to compare the predicted prevalence of high As against the observed prevalence of high As on a terrane-by-terrane scale. Because the simplest model with highest accuracy and predicted prevalence resulted in relatively high bias in some terranes of the GLAC, we chose the simplest model that minimized bias across the terranes as the 1SE model.

We further simplified the 1SE model by selecting a more parsimonious set of predictor variables by using recursive feature elimination (RFE) with the R caret package (v. 60$\left.86^{18}\right)$. The RFE method performs backwards feature selection within a 10 -fold cross validation and then ranks the predictor variables in order of importance based on their average predictive power within the model. For $\mathrm{Mn}$, we selected RFE model resulting in a combination of the highest accuracy and highest predicted prevalence. For As, we selected RFE models resulting in a combination of the highest sensitivity and highest predicted prevalence. 
We assessed the CV, 1SE, and RFE models by refitting to training data with the R package gbm (v. 2.1.5 $\left.{ }^{15}\right)$ and evaluating predictive performance with testing data. Model performance was assessed for both training and testing datasets using: sensitivity (percent of correctly classified events), specificity (percent of correctly classified nonevents), total accuracy (total percent of correct classifications), Kappa statistic ( $\mathrm{k}$; measure of inter-rater variability that accounts for randomness), the area under the receiver operator characteristic curve (AUC; indication of model performance at all classification thresholds), and predicted prevalence of high concentrations. Values of $\mathrm{k}$ range from 0 to 1 , with 0 indicating no agreement, 0.21 to 0.40 indicating fair agreement, 0.41 to 0.60 indicating moderate agreement, 0.61 to 0.8 indicating substantial agreement, and 0.81 to 0.99 indicating almost perfect agreement ${ }^{19}$. Values of ROC range from 0.5 (no predictive power) to 1 (perfect model).

For the As model, we explored the use of several methods that are commonly used to accommodate imbalanced datasets in classification modeling to improve predictive performance $^{12}$, including the following:

1. Designate 'sensitivity' as the metric to optimize during tuning

2. Downsample - randomly remove data points from majority class until the classes are balanced

3. Upsample - randomly replicate data points from minority class until classes are balanced

4. SMOTE (Synthetic Minority Oversampling Technique) - downsample data from majority class and upsample minority class. Upsampling in SMOTE interpolates data points using k-nearest neighbors.

5. Apply case weights to the data to weight minority class over majority

6. Adjust the cutoff at which predictions are considered an event

All the methods outlined above are viable options ${ }^{12}$. Literature comparisons of the different subsampling methods report variable results, with no one method standing out as being overall superior ${ }^{20,21}$.

Methods 1-5 above did not result in substantial improvement in our As model. Sensitivity for our testing data ranged from 29 to $39 \%$. The predicted proportion of events for testing data ranged from 4 to 6.5 , but the actual proportion of events is 10.7 .

By decreasing the cutoff to 0.2 for classifying the predicted probability as an event, the testing sensitivity increased from 38 to $49 \%$ and the predicted proportion of events in our testing data increased from 6.4 to 10.3 for our CV model (table S4). Although sensitivity increased substantially, the model does not reflect a sacrifice in specificity: $97 \%$ vs $94 \%$ for 0.5 and 0.2 cutoff, respectively. One caveat with using an alternative cutoff is that we accepted the risk of producing more false positives. Despite this, our model better represented some geographic areas with known high As while still adequately representing areas with low As, as illustrated in Figure 4 in the paper. 
Sensitivity and specificity were determined for Mn models based on a probability threshold of 0.5 where predicted probabilities $\geq 0.5$ were classified as an event $(\mathrm{Mn}>300$ $\mu \mathrm{g} / \mathrm{L})$ and those $<0.5$ were classified as non-events ( $\mathrm{Mn} \leq 300 \mu \mathrm{g} / \mathrm{L}$ ).

\section{Cited References}

(1) Erickson, M. L.; Yager, R. M.; Kauffman, L. J.; Wilson, J. T. Drinking Water Quality in the Glacial Aquifer System, Northern USA. Sci. Total Environ. 2019, 694, 133735. https://doi.org/10.1016/j.scitotenv.2019.133735.

(2) Nolan, B. T.; Fienen, M. N.; Lorenz, D. L. A Statistical Learning Framework for Groundwater Nitrate Models of the Central Valley, California, USA. J. Hydrol. 2015, 531, 902-911. https://doi.org/10.1016/j.jhydrol.2015.10.025.

(3) Ransom, K. M.; Nolan, B. T.; A. Traum, J.; Faunt, C. C.; Bell, A. M.; Gronberg, J. A. M.; Wheeler, D. C.; Z. Rosecrans, C.; Jurgens, B.; Schwarz, G. E.; Belitz, K.; M. Eberts, S.; Kourakos, G.; Harter, T. A Hybrid Machine Learning Model to Predict and Visualize Nitrate Concentration throughout the Central Valley Aquifer, California, USA. Sci. Total Environ. 2017, 601-602, 1160-1172. https://doi.org/10.1016/j.scitotenv.2017.05.192.

(4) Rosecrans, C. Z.; Nolan, B. T.; Gronberg, J. A. M. Prediction and Visualization of Redox Conditions in the Groundwater of Central Valley, California. J. Hydrol. 2017, 546, 341-356. https://doi.org/10.1016/j.jhydrol.2017.01.014.

(5) Erickson, M. L.; Elliott, S. M.; Christenson, C. A.; Krall, A. L. Predicting Geogenic Arsenic in Drinking Water Wells in Glacial Aquifers, North-Central USA: Accounting for Depth-Dependent Features. Water Resour. Res. 2018, 54 (12), 10,172-10,187. https://doi.org/10.1029/2018WR023106.

(6) Erickson, M. L.; Elliott, S. M.; Brown, C. J.; Stackelberg, P. E.; Ransom, K. M.; Reddy, J. E. Machine Learning Predicted Redox Conditions in the Glacial Aquifer System, Northern Continental United States. Water Resour. Res. 2021, 57, e2020WR028207. https://doi.org/10.1029/2020WR028207.

(7) Stackelberg, P. E.; Belitz, K.; Brown, C. J.; Erickson, M. L.; Elliott, S. M.; Kauffman, L. J.; Ransom, K. M.; Reddy, J. E. Machine Learning Predictions of PH in the Glacial Aquifer System, Northern USA. Groundwater 2021. https://doi.org/https://doi.org/10.1111/gwat.13063.

(8) Koch, J.; Stisen, S.; Refsgaard, J. C.; Ernstsen, V.; Jakobsen, P. R.; Højberg, A. L. Modeling Depth of the Redox Interface at High Resolution at National Scale Using Random Forest and Residual Gaussian Simulation. Water Resour. Res. 2019, 55 (2), 1451-1469. https://doi.org/10.1029/2018WR023939.

(9) Tesoriero, A. J.; Gronberg, J. A.; Juckem, P. F.; Miller, M. P.; Austin, B. P. Predicting Redox-Sensitive Contaminant Concentrations in Groundwater Using Random Forest Classification. Water Resour. Res. 2017, 53 (8), 7316-7331. https://doi.org/10.1002/2016WR020197. 
(10) Breiman, L. Statistical Modeling: The Two Cultures. Stat. Sci. 2001, 16 (3), 199-215. https://doi.org/10.1214/ss/1009213726.

(11) Elith, J.; Leathwick, J. R.; Hastie, T. A Working Guide to Boosted Regression Trees. J. Anim. Ecol. 2008, 77 (4), 802-813. https://doi.org/10.1111/j.13652656.2008.01390.x.

(12) Kuhn, M.; Johnson, K. Applied Predictive Modeling; Springer - New York, 2013.

(13) Ayotte, J. D.; Nolan, B. T.; Gronberg, J. A. Predicting Arsenic in Drinking Water Wells of the Central Valley, California. Environ. Sci. Technol. 2016, 50, 7555-7563. https://doi.org/10.1021/acs.est.6b01914.

(14) Ayotte, J. D.; Medalie, L.; Qi, S. L.; Backer, L. C.; Nolan, B. T. Estimating the HighArsenic Domestic-Well Population in the Conterminous United States. Environ. Sci. Technol. 2017, acs.est.7b02881. https://doi.org/10.1021/acs.est.7b02881.

(15) Ridgeway, G. gbm: Generalized Boosted Regression Models. R Package Version 2.1. https://cran.r-project.org/web/packages/gbm/gbm.pdf.

(16) R Development Core Team. R: A Language and Environment for Statistical Computing. R Foundation for Statistical Computing: Vienna, Austria 2018.

(17) Wenger, S. J.; Olden, J. D. Assessing Transferability of Ecological Models: An Underappreciated Aspect of Statistical Validation. Methods Ecol. Evol. 2012, 3 (2), 260-267. https://doi.org/10.1111/j.2041-210X.2011.00170.x.

(18) Kuhn, M. Caret: Classification and Regression Training. R Package Version 6.0-86. 2020.

(19) Viera, A. J.; Garrett, J. M. Understanding Interobserver Agreement: The Kappa Statistic. Fam. Med. 2005, 37 (5), 360-363. https://doi.org/Vol. 37, No. 5.

(20) Prati, R. C.; Batista, G. E. A. P. A.; Silva, D. F. Class Imbalance Revisited: A New Experimental Setup to Assess the Performance of Treatment Methods. Knowl. Inf. Syst. 2015, 45, 247-270. https://doi.org/10.1007/s10115-014-0794-3.

(21) Van Hulse, J.; Khoshgoftaar, T. M.; Napolitano, A. Experimental Perspectives on Learning from Imbalanced Data. In Proceedings of the 24th International Conference on Machine Learning,; Corvallis, OR, 2007; p 8 p. 
A
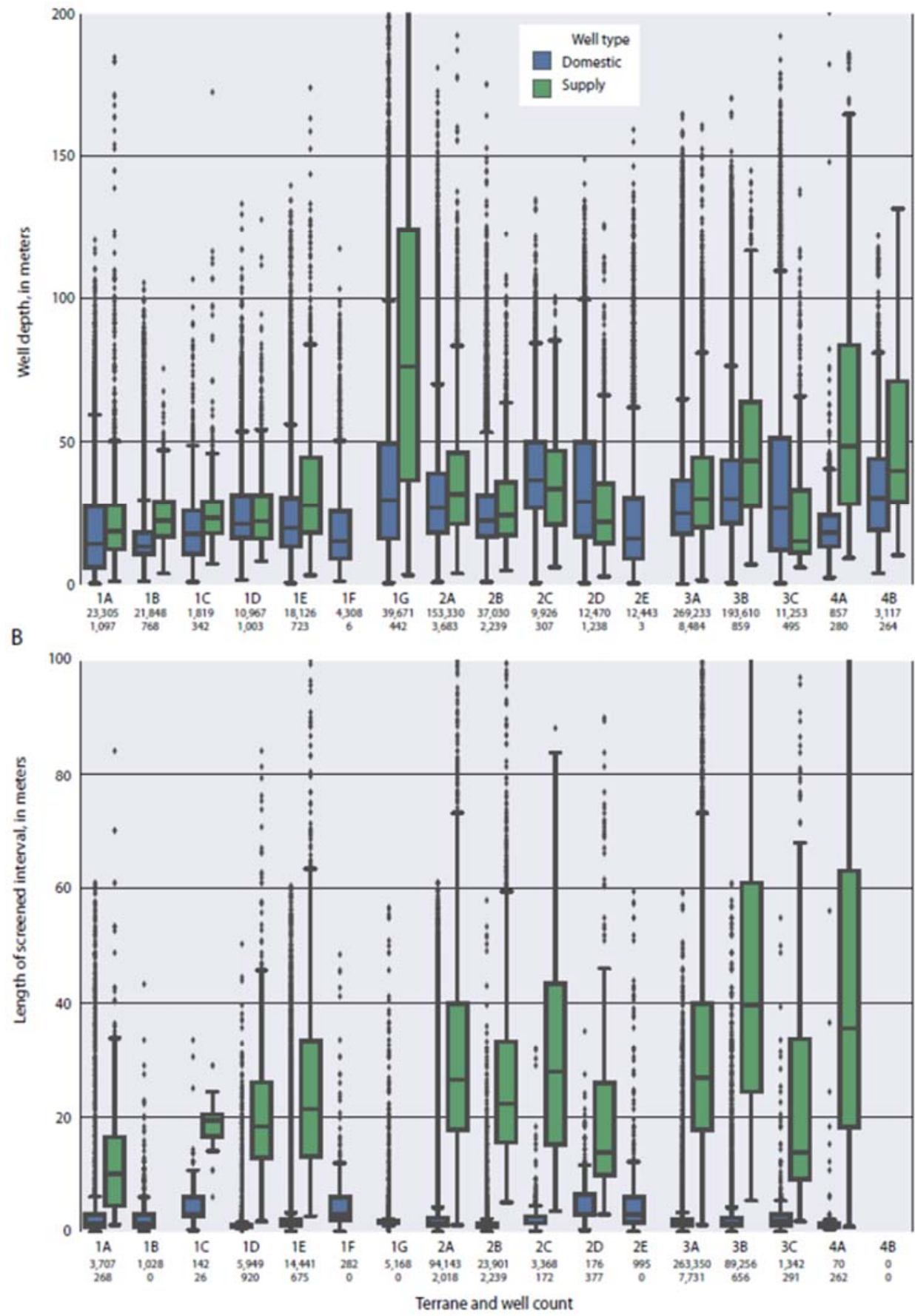

\section{EXPLANATION}

Individual sample above the 90 th percentile

90 th percentile

75 th percentile

50 th percentile (median)

25 th percentile

1 10th percentile

Figure S1. Figure S1 Well construction box plots, domestic and public wells by terrane (fig. 1); (A) well depth, (B) length of screened interval. Number of samples for domestic, public supply below terrane labels (Figure SI-1 in Erickson et al., 2019). 


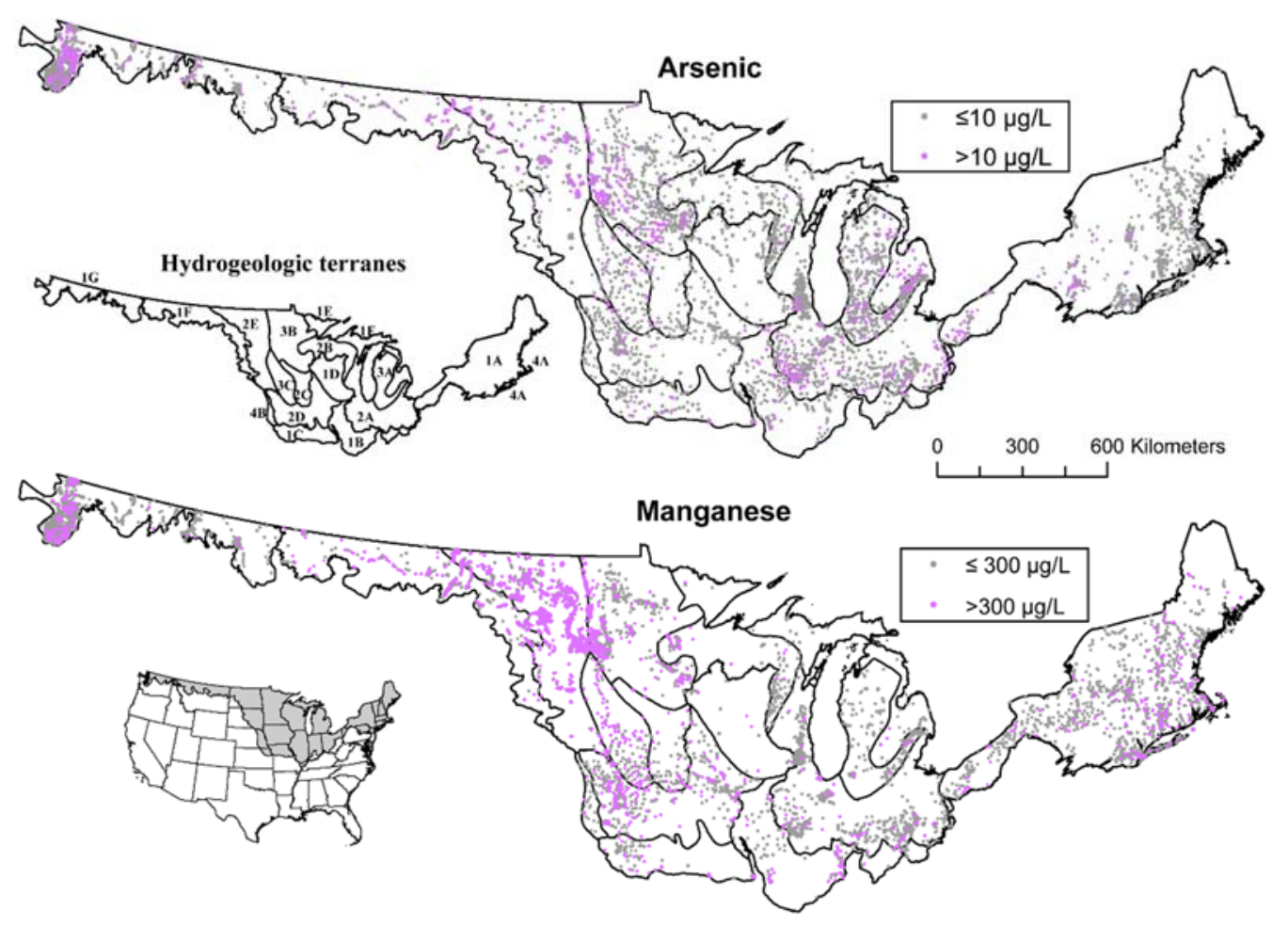

Figure S2. Response variable data distribution for arsenic and manganese in the study area. 
Soil $\mathrm{C}$ horizon arsenic concentration

Predicted $\mathrm{pH}$ at domestic water supply depths

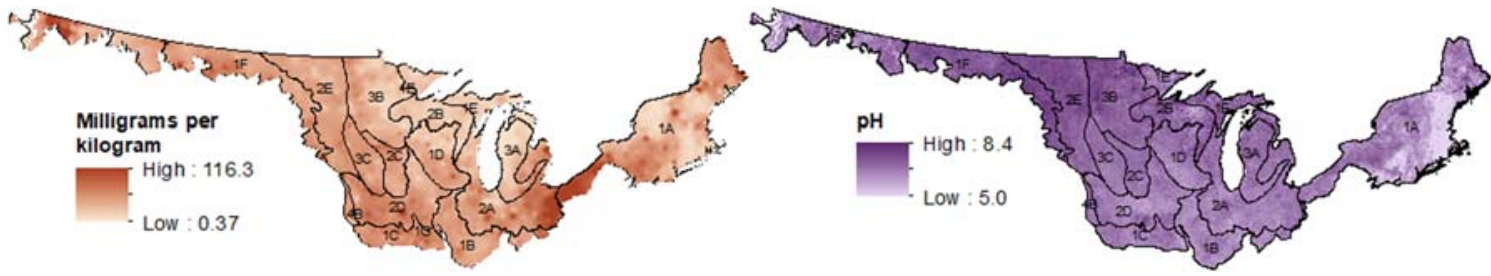

Coarse sediment thickness of glacial deposits

Predicted probability of high iron at domestic
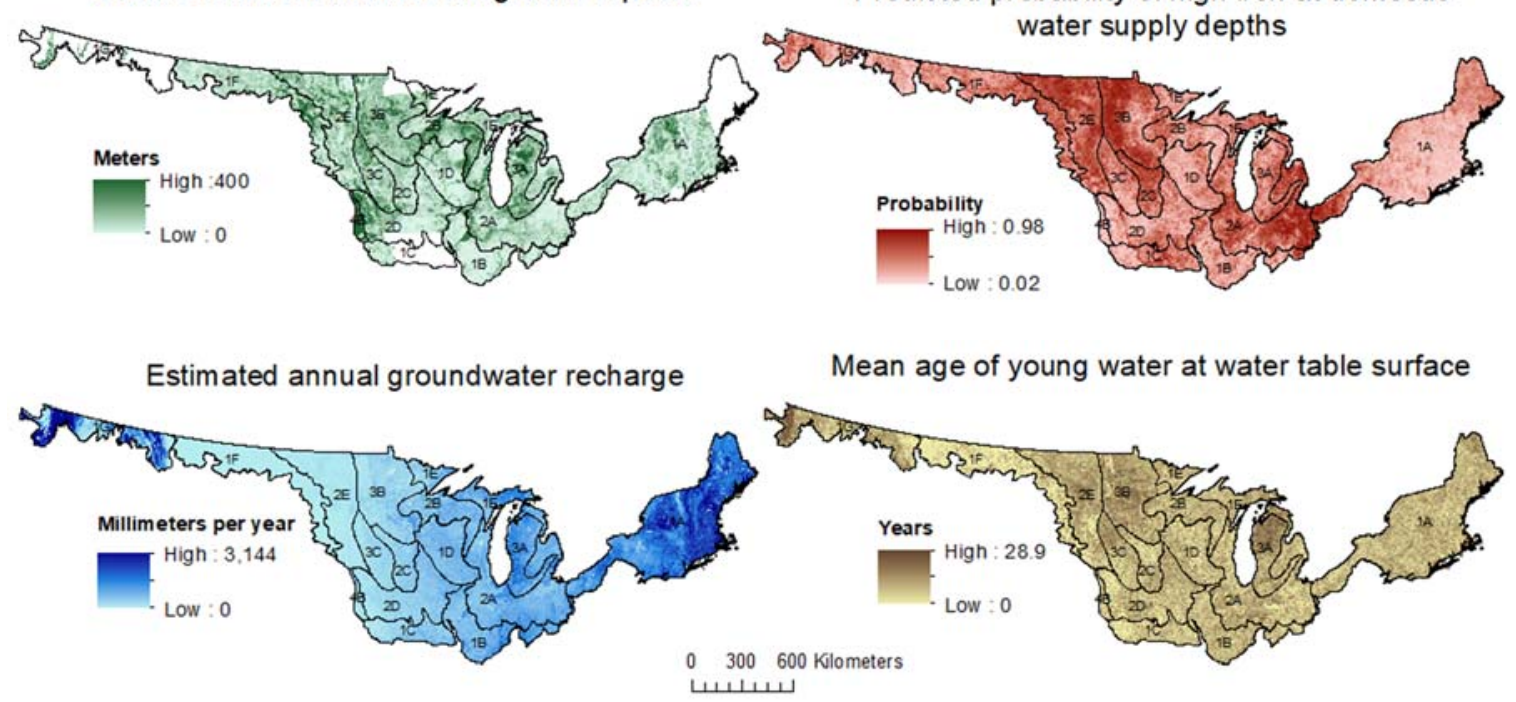

Figure S3. Example maps of selected predictor variables. 


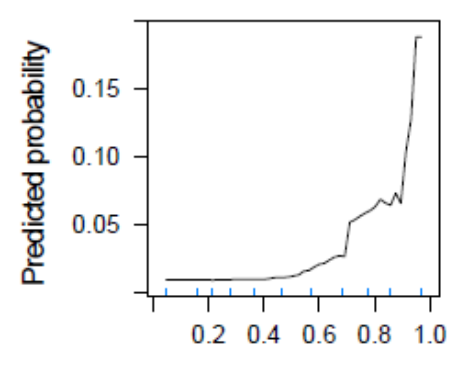

ProbHighFe100
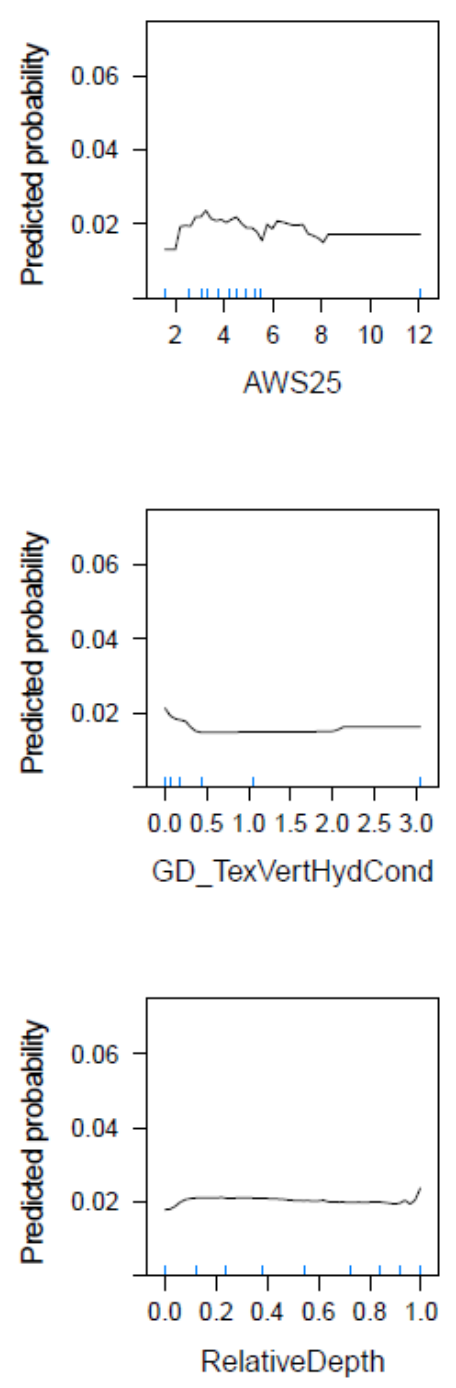

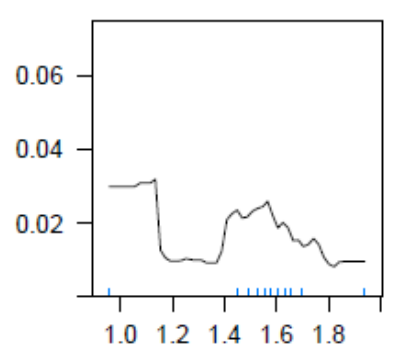

SoilBulkDens
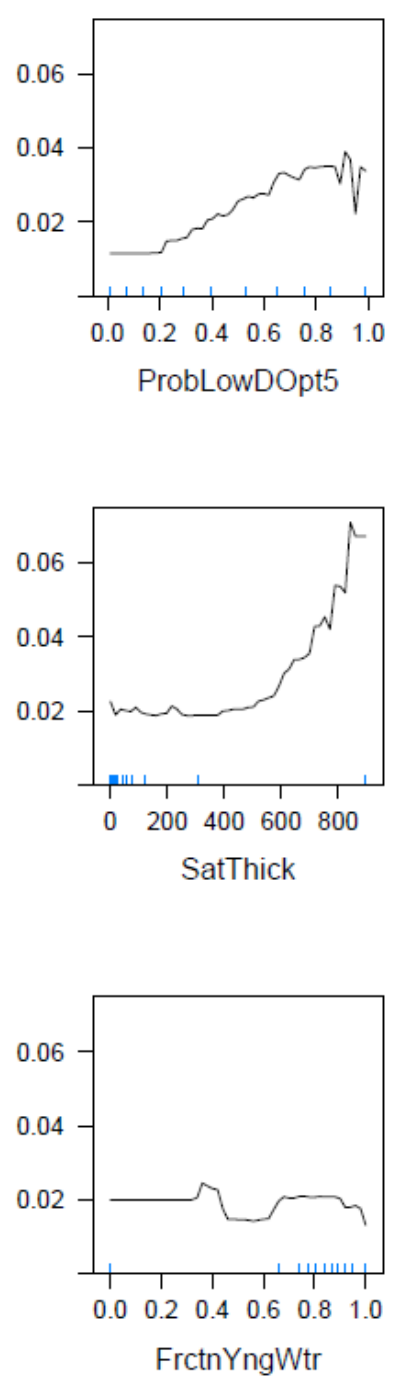
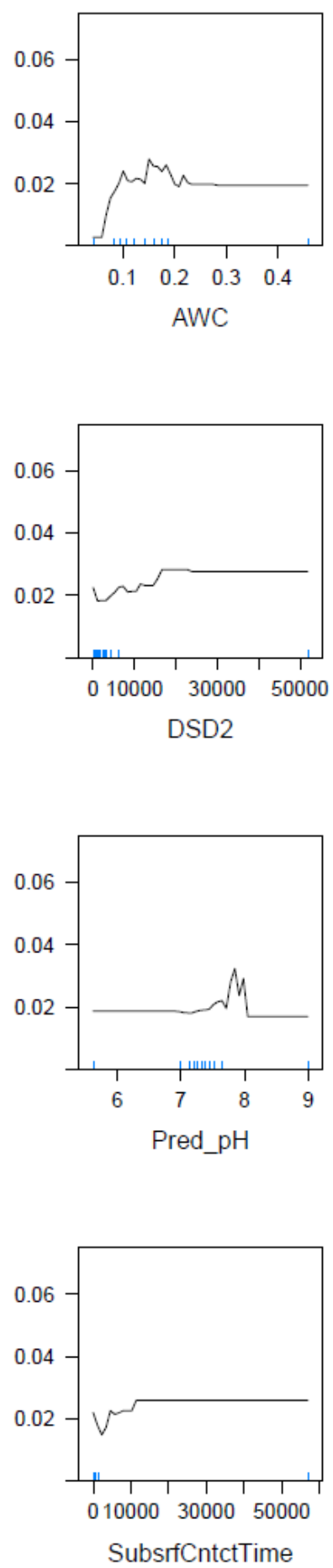

Figure S4. Partial dependence plots (PDPs) for As $>10 \mu \mathrm{g} / \mathrm{L}$ model, predictor variables with relative influence $1-12$, with data deciles denoted with tick marks. Units provided in Table S3. PDPs display the average model prediction as the variable of interest is varied over a range of values. The relationships depicted by PDPs may not be accurately represented where data are sparse. 

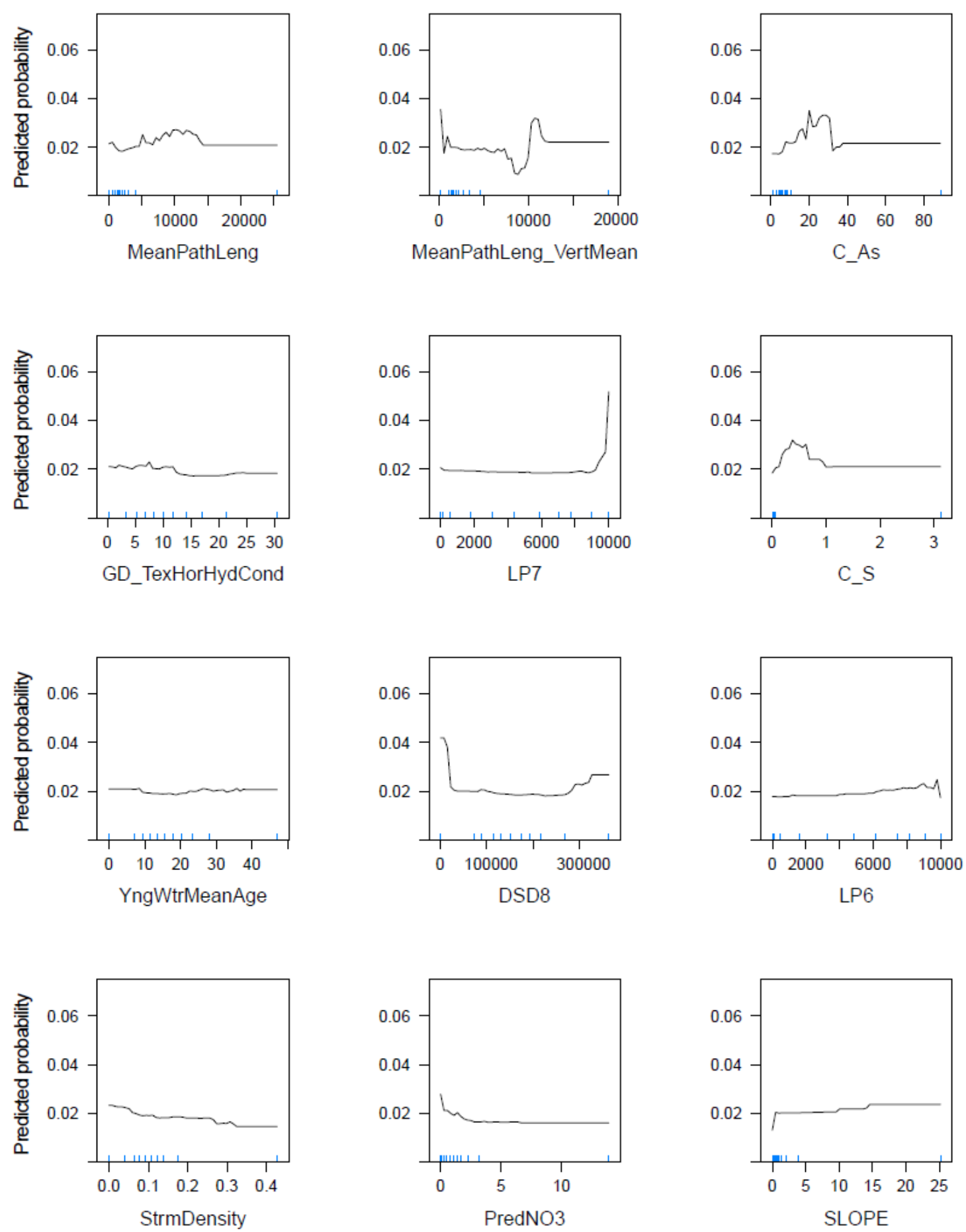

Figure S5. Partial dependence plots for As $>10 \mu \mathrm{g} / \mathrm{L}$ model, predictor variables with relative influence $13-24$, with data deciles denoted with tick marks. Units provided in Table S3. PDPs display the average model prediction as the variable of interest is varied over a range of values. The relationships depicted by PDPs may not be accurately represented where data are sparse. 

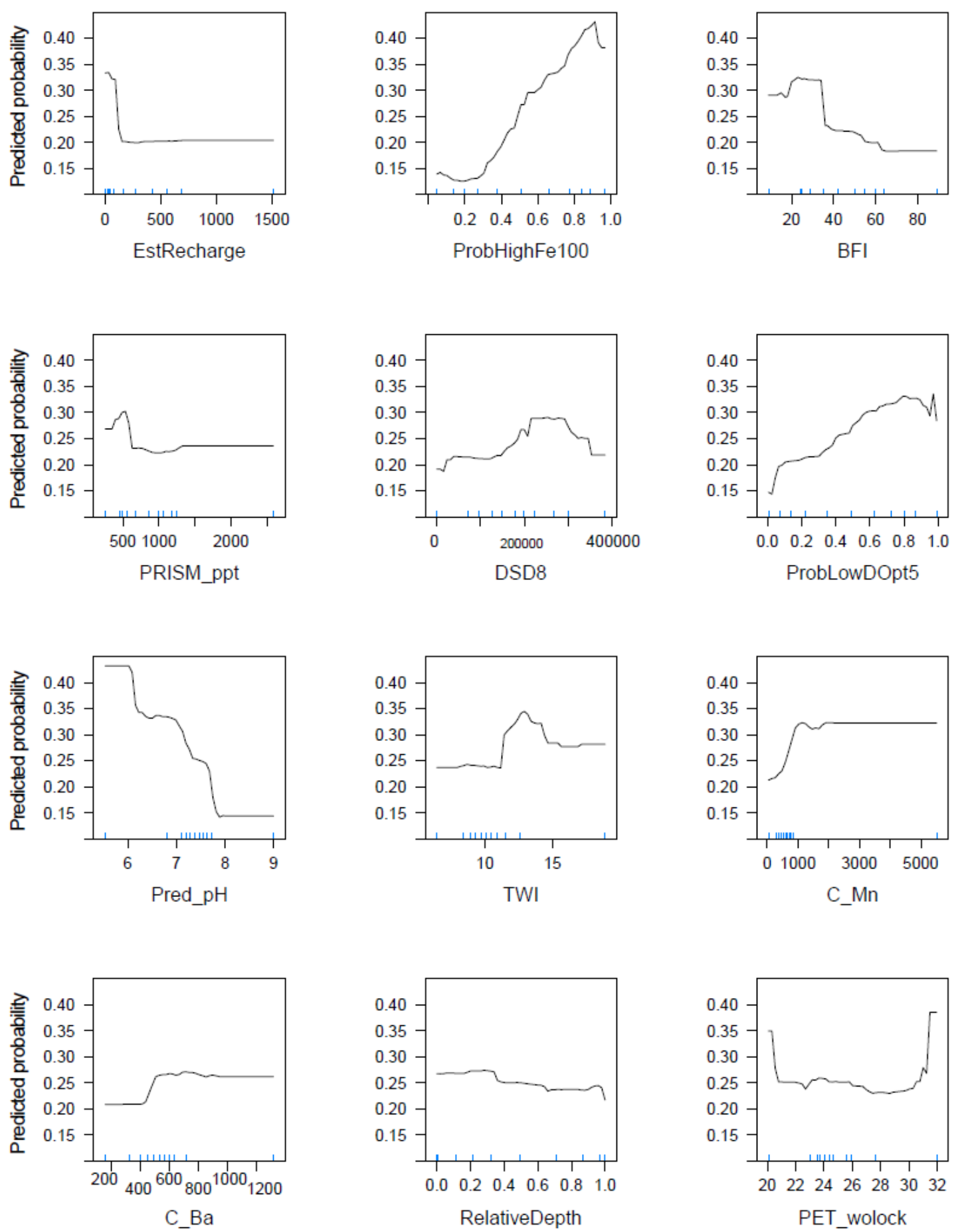

Figure S6. Partial dependence plots for $\mathrm{Mn}>300 \mu \mathrm{g} / \mathrm{L}$ model, predictor variables with relative influence $1-12$, with data deciles denoted with tick marks. Units provided in Table S3. PDPs display the average model prediction as the variable of interest is varied over a range of values. The relationships depicted by PDPs may not be accurately represented where data are sparse. 

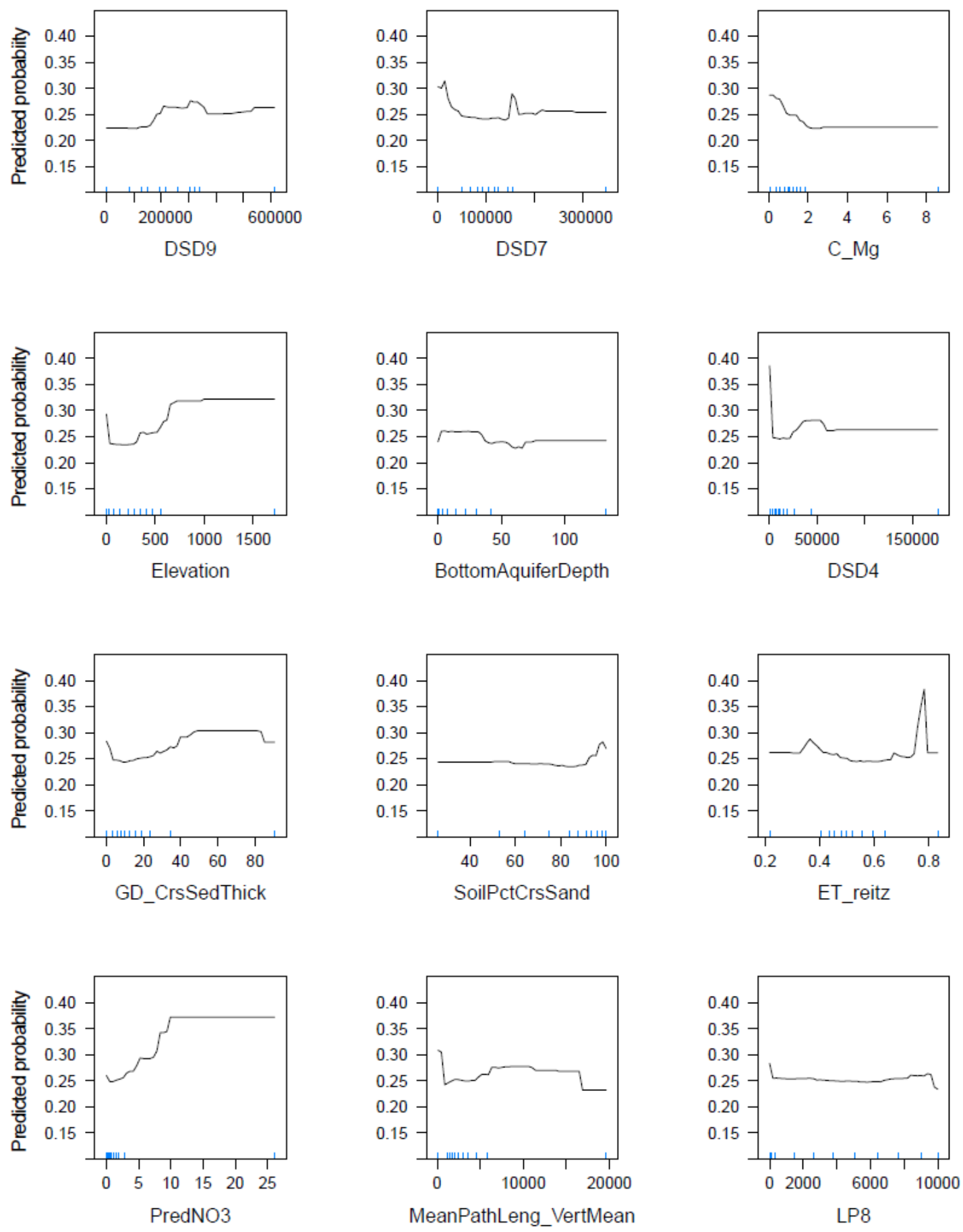

Figure S7. Partial dependence plots for $\mathrm{Mn}>300 \mu \mathrm{g} / \mathrm{L}$ model, predictor variables with relative influence $13-24$, with data deciles denoted with tick marks. Units provided in Table S3. PDPs display the average model prediction as the variable of interest is varied over a range of values. The relationships depicted by PDPs may not be accurately represented where data are sparse. 

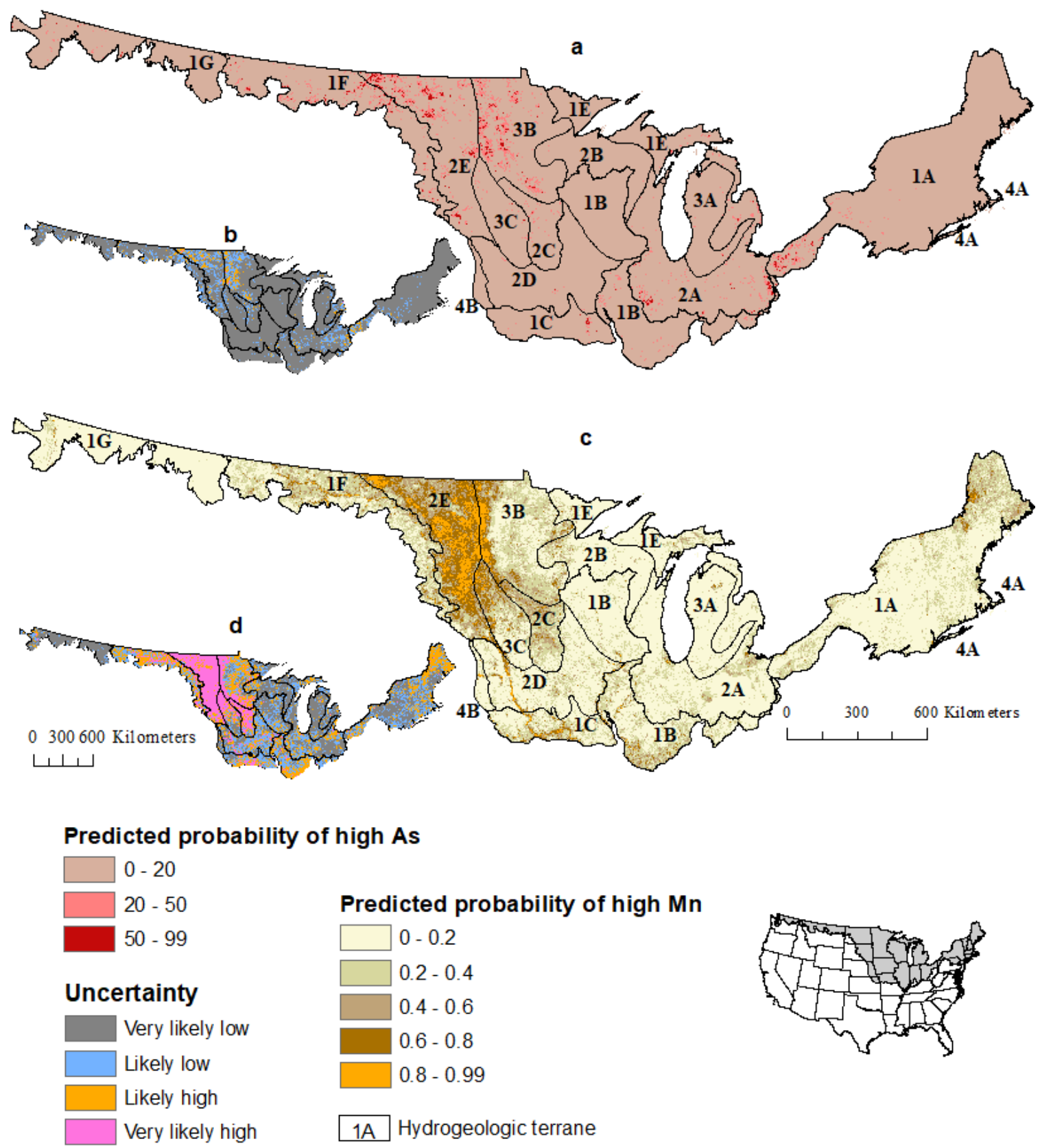

Figure S8. Modeled probability of high As indicated by $A s>10 \mu \mathrm{g} / \mathrm{L}$ at (a) Domestic well depth surface (Fig.1, table S1), and (b) uncertainty category based on prediction interval (Table S6); Modeled probability of high Mn indicated by $\mathrm{Mn}>300 \mu \mathrm{g} / \mathrm{L}$ at (c) Domestic well depth surface (Fig. 1, table S1), and (d) uncertainty category (Table S6). 